\title{
Laryngeal Hemorrhage, CTCAE
}

National Cancer Institute

\section{Source}

National Cancer Institute. Laryngeal Hemorrhage, CT CAE. NCI Thesaurus. Code C56545.

A disorder characterized by bleeding from the larynx. 